\title{
Custirsen Sodium
}

National Cancer Institute

\section{Source}

National Cancer Institute. Custirsen Sodium. NCI Thesaurus. Code C1055.

The eicosasodium salt of a mixed-backbone antisense olig odeoxynucleotide with chemosensitizing properties. Custirsen inhibits testosterone-repressed prostate message-2 (TRPM-2). Administration of custirsen abrogates the anti-apoptotic effect of TRPM-2, thereby sensitizing cells to chemotherapy and resulting in tumor cell death.

TRPM-2 is an anti-apoptotic clusterin that is overexpressed by prostate cancer cells and is associated with chemoresistance. 